\title{
PROCESS AND SYSTEMS 'Fast-track' pathway for resectable pancreatic cancer: barriers and facilitators to implementation across a regional network
}

\author{
Authors: Basil Yannoulias, ${ }^{A}$ Mark Exworthy ${ }^{B}$ and Keith J Roberts ${ }^{c}$
}

\section{Introduction}

The National Institute for Health and Care Excellence guidelines recommend a 'fast-track' approach to avoid preoperative biliary drainage (PBD) when treating resectable pancreatic cancer. For reasons not yet known, there is variable uptake of this approach across the UK. A 'fast-track' pathway which avoids PBD was introduced in University Hospitals Birmingham NHS Foundation Trust (UHB) and referring centres in 2015.

\section{Methodology}

Eleven semi-structured interviews were conducted with members of the hepatobiliary multidisciplinary team (MDT) in UHB and referring centres. Barriers and facilitators to pathway implementation were assessed.

Results

Facilitators underpinning implementation were collaboration between stakeholders, clinical leadership and careful coordination of referrals. Barriers to implementation included clinician opposition and increased workload. Barriers were mitigated through phased implementation and the appointment of dedicated staff.

Conclusion

Future work may focus on exploring contextual factors in other tertiary centres and evaluating the emotional impact of 'fast-tracked' versus delayed surgery in patients with resectable pancreatic cancer.

KEYWORDS: fast-track surgery, pancreatic cancer, care pathways

DOI: $10.7861 /$ fhj.2021-0042

\section{Introduction}

For patients with pancreatic cancer to realise the best chance for cure, they must undergo surgery and receive multiagent

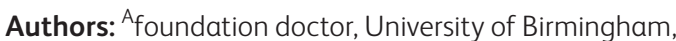
Birmingham, UK; ${ }^{B}$ professor of health policy and management, University of Birmingham, Birmingham, UK; ${ }^{C}$ consultant liver transplant, hepatobiliary and pancreatic surgeon and honorary reader, University of Birmingham, Birmingham, UK chemotherapy. ${ }^{1,2}$ Delays to surgery and/or complications in the perioperative period reduce the chances for both curative surgery and receipt of chemotherapy. ${ }^{3}$ Therefore, pathways to optimise the chances for patients to undergo these treatments are essential. Most patients who undergo surgery for pancreatic cancer develop jaundice as their presenting complaint. Jaundice has been thought to increase the risk of perioperative and postoperative complications, so preoperative biliary drainage (PBD) became standard practice to improve jaundice prior to surgery. ${ }^{4}$ Level 1 evidence supports a direct 'fast track' to surgery approach while the traditional approach was to perform PBD. ${ }^{5}$ Complications due to PBD include pancreatitis, biliary tract infection and bleeding; the process of PBD delays surgery and it is not uncommon for repeated attempts to be needed, which further delays surgery. ${ }^{6}$ The approach to avoid PBD thus reduces perioperative complications and achieves early surgery where more patients undergo cancer resection. ${ }^{7}$ Consequently, the National Institute for Health and Care Excellence (NICE) recommends avoiding PBD for suitable patients. ${ }^{8}$ However, despite the benefits and national guidance, the majority of pancreatic cancer networks across the UK have yet to implement successful 'fast-track' pathways. ${ }^{9}$

The first fast-track pathway in the UK which avoids PBD prior to referral to tertiary care was introduced at University Hospitals Birmingham NHS Foundation Trust (UHB) and referring centres in 2015. ${ }^{7}$ Patients' eligibility for the pathway was determined by meeting clinical criteria and a minimum World Health Organization (WHO) performance status score; the pathway and eligibility criteria for patients are summarised in Fig 1 and Table $1 .^{10}$

The pathway was implemented with considerable success. From 01 August 2015 to 31 July 2016, 145 patients underwent attempted resection in UHB, of which 52 were excluded at the point of referral on clinical grounds. ${ }^{7}$ Throughout the 12 -month period, 61 and 32 patients underwent resection with and without PBD, respectively. ${ }^{7}$ It was found that the time from initial computed tomography (CT) to surgery was significantly reduced in the group that did not receive PBD (16 days versus 65 days; $\mathrm{p}<0.0001)$. ${ }^{7}$ In addition, more patients underwent resection in the group without PBD compared with the PBD group (31/32 versus $46 / 61$, respectively; $p=0.009){ }^{7}$ At the time of writing, over $80 \%$ of patients referred to UHB with pancreatic malignancy are treated via the fast-track pathway.

The pathway also led to significant financial savings. Roberts et al highlighted the need for a dedicated pathway nurse to 


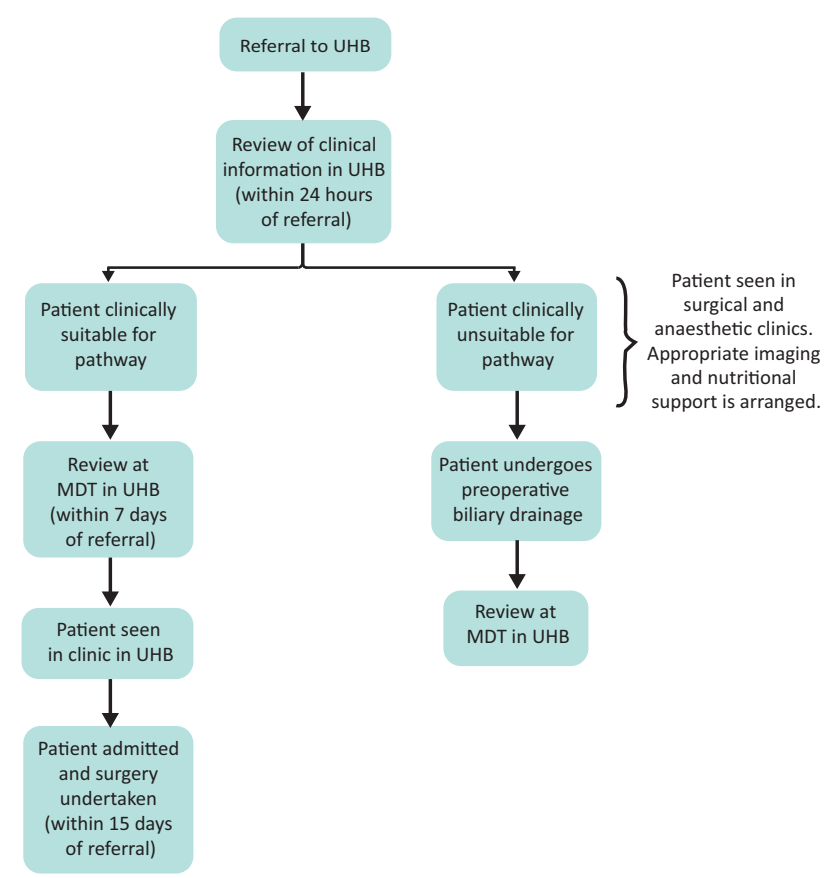

Fig 1. Fast-track pathway summary. $M D T=$ multidisciplinary team; $U H B=$ University Hospitals Birmingham NHS Foundation Trust.

coordinate the referral process. ${ }^{7}$ In order to fund this position, a $£ 50,000$ award from Pancreatic Cancer UK as well as a $£ 50,000$ grant from University Hospitals Birmingham Charity were awarded. ${ }^{11}$ Given that approximately $£ 3,200$ is saved for each patient treated with fast-track surgery, the costs of the project were recuperated within a year of implementation. ${ }^{11}$

\begin{tabular}{|c|c|c|}
\hline Variable & Inclusion criteria & Exclusion criteria \\
\hline $\begin{array}{l}\text { Bilirubin at the time } \\
\text { of surgery }\end{array}$ & $\begin{array}{l}\text { Likely to be }<450 \\
\mu \mathrm{mol} / \mathrm{L}\end{array}$ & $\begin{array}{l}\text { Likely to be }>450 \\
\mu \mathrm{mol} / \mathrm{L}\end{array}$ \\
\hline Venous involvement & $\begin{array}{l}\text { Involvement } \\
\text { of SMV and PV } \\
\text { with potential } \\
\text { for surgical } \\
\text { reconstruction }\end{array}$ & $\begin{array}{l}\text { Complete occlusion } \\
\text { of SMV/PV and/or } \\
\text { without possibility } \\
\text { for surgical } \\
\text { reconstruction }\end{array}$ \\
\hline $\begin{array}{l}\text { Involvement of } \\
\text { superior mesenteric } \\
\text { artery or coeliac axis }\end{array}$ & None & All \\
\hline Biliary sepsis & None & All \\
\hline Renal dysfunction & $\begin{array}{l}\text { Able to be } \\
\text { corrected with } \\
\text { short course of } \\
\text { fluid therapy }\end{array}$ & $\begin{array}{l}\text { Not able to be } \\
\text { corrected with short } \\
\text { course of fluid } \\
\text { therapy }\end{array}$ \\
\hline $\begin{array}{l}\text { World Health } \\
\text { Organization } \\
\text { performance status }\end{array}$ & $<2$ & $\geq 2$ \\
\hline
\end{tabular}

Our study aims to explore barriers and facilitators to the implementation of the fast-track pathway in a tertiary hepatobiliary unit, UHB, and its referring centres using qualitative methods.

\section{Methodology}

The views of hepatobiliary multidisciplinary team (MDT) members in UHB and referring centres were explored through semi-structured interviews. Semi-structured interviews enable participants to fully express themselves verbally, while allowing the researcher to control the focus of each interview. ${ }^{12}$ The interviewer adhered to a topic guide (Box 1 ) to ensure key topics were addressed, although flexibility in its use allowed emerging themes to be explored as they arose. ${ }^{13}$ The guide was refined following initial piloting with a member of the hepatobiliary MDT in UHB.

For recruitment, a snowball method was used whereby an established contact in UHB provided email addresses of hepatobiliary MDT members both within UHB and in referring centres who would be suitable for the study. Interviewees were sampled for maximum variation across a range of professions, which ensured that the views of a diversity of professionals were considered. Recruitment continued until data saturation had been achieved, whereby no new analytical themes emerged, at which point 11 clinicians had been interviewed (Table 2). Interviews were carried out between 18 February 2020 and 16 March 2020, and each lasted between 21 and 32 minutes; these were recorded using a digital voice recorder and transcribed verbatim. Qualitative analysis was in the form of a thematic analysis using Braun and

Box 1. Interview topic guide

\section{Introduction}

Introduce yourself.

State that interview will last around 30 minutes.

Check that participant is happy to start.

\section{Question 1}

Can you tell me about your own involvement in the delivery of pancreatic cancer services?

\section{Question 2}

A fast-track pathway for pancreatic cancer surgery was implemented as a pilot in University Hospitals Birmingham NHS Foundation Trust in 2015. How familiar are you with this work?

\section{Question 3}

Do you feel your own practice has changed in your centre as a result of the fast-track pathway?

Probe

How has practice changed?

\section{Question 5}

What do you think were the main barriers to implementing the pathway at your centre?

\section{Question 6}

What factors do you feel helped in the implementation of the pathway at your centre?

\section{Question 7}

Do you feel the pathway is sustainable in the long term?

\section{Question 8}

Is there anything else you would like to say?

Thank participant. 
Table 2. Interviewee characteristics

\begin{tabular}{|c|c|c|c|c|c|}
\hline $\begin{array}{l}\text { Interviewee } \\
\text { number }\end{array}$ & Interviewee role & $\begin{array}{l}\text { Method of } \\
\text { recruitment }\end{array}$ & $\begin{array}{l}\text { Base NHS } \\
\text { trust }\end{array}$ & $\begin{array}{l}\text { Length of } \\
\text { interview, minutes }\end{array}$ & $\begin{array}{l}\text { Interview } \\
\text { modality }\end{array}$ \\
\hline 1 & Consultant hepatobiliary surgeon & Snowball method & UHB & 31 & Face-to-face \\
\hline 2 & Consultant hepatobiliary surgeon & Established contact & UHB & 25 & Face-to-face \\
\hline 3 & Hepatobiliary clinical nurse specialist & Snowball method & UHB & 28 & Face-to-face \\
\hline 4 & Consultant hepatobiliary surgeon & Snowball method & UHB & 24 & Face-to-face \\
\hline 5 & Hepatobiliary clinical nurse specialist & Snowball method & UHB & 22 & Face-to-face \\
\hline 6 & Hepatobiliary clinical nurse specialist & Snowball method & Referrer & 26 & Telephone \\
\hline 7 & Consultant gastroenterologist & Snowball method & Referrer & 30 & Telephone \\
\hline 8 & Consultant upper gastrointestinal surgeon & Snowball method & Referrer & 32 & Telephone \\
\hline 9 & Consultant medical oncologist & Snowball method & UHB & 21 & Telephone \\
\hline 10 & Hepatobiliary clinical nurse specialist & Snowball method & Referrer & 27 & Telephone \\
\hline 11 & Hepatobiliary clinical nurse specialist & Snowball method & Referrer & 21 & Telephone \\
\hline
\end{tabular}

Clarke's six-phase method. ${ }^{14}$ Emergent themes were categorised as facilitators (factors considered to enable the implementation of the pathway) and barriers (factors to be overcome in order to successfully implement the pathway).

\section{Ethics}

Ethical approval for the study was obtained from the University of Birmingham Internal Research and Ethics Committee prior to data collection.

\section{Results}

\section{Facilitator: collaboration between centres}

A key factor behind implementation according to interviewees has been the collaborative atmosphere between referring centres and $U H B$, which has been fostered since the introduction of the pathway in 2015. It was noted that UHB staff frequently visit

\section{Box 2. Tips at a glance for implementing a fast-} track pathway

> Clinical leaders should regularly meet with referrers to champion the benefits of fast-track surgery to clinicians and managers, discuss cases and gain feedback.

- Ensure dedicated staff are available to manage the workload associated with fast-track referrals and keep patients wellinformed throughout their treatment journey. This may require additional funding, but costs will be rapidly offset by financial benefits of earlier surgery.

Ensure adequate theatre space is available to accommodate fast-track referrals.

> 'Start small' in a few referring centres and learn from experience to resolve organisational challenges.

> Consider providing referrers with a defined protocol to ensure that the correct information is provided with each referral. referring hospitals in order to discuss cases and gain feedback: interviewee 8:

From the start they have made a real effort to attend our MDTs, give feedback on cases, suggest improvements, ask what we think.

Similarly, referring clinicians noted that the availability of nurses in UHB to receive enquiries or questions regarding 'fast-track' patients has lessened their anxieties when making referrals; interviewee 6:

\section{Being able to talk to my nurse equivalent has helped.}

\section{Facilitator: clinical leadership}

Clinical leadership, particularly from consultants in UHB, was seen to facilitate the implementation of the pathway. Consultant clinicians in UHB visited trusts individually to increase awareness. Clinical leadership was also used to gain buy-in from hospital managers within UHB, who were made aware of the logistical advantages of earlier surgery for resectable pancreatic cancer patients, and from clinical nurse specialists, who play a major role in coordinating referrals and communicating with patients. Interviewee 2:

I think within 3 or 6 months, we'd been to all the referring trusts.

To ensure that eligible patients were not missed, clinicians in referring centres led subsequent efforts to make new staff aware of the pathway and the referral protocol; interviewee 8:

We make sure that we keep advertising it to new staff that come in.

\section{Facilitator: careful coordination of referrals}

In order to accommodate fast-track referrals, several organisational changes were made within UHB. These include ensuring availability of theatre space, which was achieved by blocking out two theatre slots a week for fast-track patients. If slots remain unfilled, a degree of flexibility allowed other patients to be 
rescheduled to these sessions. This process was aided by the fact that hepatobiliary surgeons in UHB do not have named operating lists, rather, the surgical team coordinate schedules among themselves to ensure that each slot is covered; interviewee 2 :

We've found it quite easy to do the operating theatres because we're a big team and we don't have named lists.

On the other hand, interviewees noted that patients sometimes expressed a feeling of being rushed, with little time to mentally process their 'fast-tracked' surgery. In order to ensure patients remained well-informed, effective communication with patients was prioritised and facilitated largely through communication between clinical nurse specialists in UHB and referring centres.

\section{Barrier: building clinician acceptance}

Some interviewees predicted that a barrier to implementation at other specialist sites would be opposition shown by some clinicians to the early surgery approach, due to the emerging practice of neoadjuvant therapy (NAC) in pancreatic cancer, whereby patients are treated with chemotherapy prior to surgery. ${ }^{15}$ Avoiding PBD among jaundiced patients who proceed with chemotherapy is not possible whatsoever. Interviewee 2:

Some surgeons lean towards the neoadjuvant approach. Is that better or not? We need high quality randomised data for that.

Clinician acceptance of the pathway among referrers was aided by a 'soft launch' in only two referring centres, which allowed time to overcome organisational issues. For example, the bilirubin threshold for patient eligibility was gradually increased and a defined protocol to which referrers could work was introduced to help avoid missed appointments and ensure the correct information was supplied with each patient; interviewee 5 :

We now have tick boxes to help sort clinical information for each case.

\section{Barrier: increased work burden}

The large work burden in UHB resulting from the fast-track pathway was mentioned, particularly for the clinical nurse specialists who play a major role in coordinating the referral process. Interviewee 3 :

If you have more than two referrals on the same day, that takes a lot of time and there are still other patients.

Following the appointment of a dedicated fast-track coordinator, interviewees found that the workload became more manageable. ${ }^{9}$ They suggested that having a dedicated coordinator has allowed more time for other clinical duties.

\section{Discussion}

Collaboration between stakeholders in UHB and referring centres, which is largely facilitated by clinical nurse specialists who are able to manage the communication between referring units, UHB and patients, was seen as a key facilitator behind the successful implementation of the pathway. In addition, the pathway relies on its clinical leaders to 'sell' the benefits to stakeholders and ensure ongoing referrer engagement, particularly among new staff to ensure that they are made aware of the referral process for eligible patients.
Given that the fast-track pathway described is the first to be implemented in the UK, data from other centres is not available; however, when published, data from the Receipt of Curative Resection or Palliative Care for Hepatopancreaticobiliary Tumours (RICOCHET) study will elucidate national variation in treatment of pancreatic malignancies. ${ }^{16}$ The findings of our study echo the assertion by Best et al that successful system transformation relies on engaging physicians, balancing designated with distributed leadership and learning from shortcomings. ${ }^{17}$ Clinical leaders of the fast-track pathway regularly visited referring centres to engage referrers, who were then able to champion the pathway within their own centres. These visits also allowed feedback to be passed to UHB to help facilitate ongoing improvements, such as the introduction of a defined protocol for referrals. Best et al also point out that change efforts should be patient centred; improving outcomes for patients with pancreatic cancer is the primary reason for the pathway, although the emotional effects of 'fast-tracked' versus delayed surgery remain to be formally assessed and this presents a potential avenue for future qualitative work. ${ }^{15}$

The need to build clinician acceptance was noted by some interviewees at UHB; other centres may also find that phased implementation of the pathway may be beneficial as organisational challenges are resolved. In addition, our study highlights the need for randomised controlled trials comparing NAC with fast-track surgery, such that patients in the control group have the advantage of avoiding PBD.

Furthermore, practical factors (such as flexible theatre space and the appointment of dedicated staff) ensured sustainability of the pathway in UHB and referring centres, suggesting that other tertiary centres would need to tailor the fast-track pathway to meet local needs. To assist in the wider uptake of the pathway, future research may, therefore, focus on gaining an understanding of contextual factors in other tertiary centres and their referrers.

\section{Strengths and limitations}

This study is the first to qualitatively consider the factors underpinning the implementation of a fast-track service for resectable pancreatic cancer. The key strength of the study is that it provides accounts from clinicians representing a range of professions in both UHB and referring centres. This range of viewpoints ensured that data saturation was achieved. As an evaluation of a regional service, the results are not directly transferable to other contexts; however, the insights gained should guide other centres to implement a fast-track pathway to reflect recent NICE recommendations. ${ }^{8}$

The study is limited in that patients were not interviewed; instead, clinicians provided insight into the patient experience, including the perceived emotional impact on patients due to rapid diagnosis and early surgery. In addition, a drawback of a snowball sampling strategy is the potential for gatekeeper bias. ${ }^{18}$ The initial contacts from UHB were leaders of the fast-track pathway and, therefore, may have suggested other clinicians who favoured the pathway; however, the input provided by these individuals was valuable to the study.

\section{Conclusion}

The study suggests that the successful implementation of the fasttrack pathway was underpinned by close collaboration between clinicians in UHB and referring centres, supported by dedicated 
staff to coordinate referrals. The efforts of clinical leaders to champion the benefits of the pathway built clinician acceptance and promoted ongoing engagement from referrers. These insights provide a framework to guide implementation of fast-track pancreatic cancer surgery services by other centres in the UK.

\section{References}

1 Conroy T, Hammel P, Hebbar M et al. FOLFIRINOX or gemcitabine as adjuvant therapy for pancreatic cancer. New Engl J Med 2018;379:2395-406.

2 Neoptolemos JP, Palmer DH, Ghaneh P et al. Comparison of adjuvant gemcitabine and capecitabine with gemcitabine monotherapy in patients with resected pancreatic cancer (ESPAC-4): a multicentre, open-label, randomised, phase 3 trial. Lancet 2017:389:1011-24.

3 Sanjeevi $S$, Ivanics $T$, Lundell $L$ et al. Impact of delay between imaging and treatment in patients with potentially curable pancreatic cancer. Br J Surg 2016;103:267-75.

4 van der Gaag NA, Kloek J], de Castro SM et al. Preoperative biliary drainage in patients with obstructive jaundice: history and current status. J Gastrointest Surg 2009;13:814-20.

5 van der Gaag NA, Rauws EA, van Eijck CH et al. Preoperative biliary drainage for cancer of the head of the pancreas. N Engl J Med 2010;362:129-37.

6 Pandé R, Hodson J, Murray A et al. Evaluation of the clinical and economic impact of delays to surgery in patients with periampullary cancer. BJS Open 2019;3:476-84.

7 Roberts KJ, Prasad P, Steele $Y$ et al. A reduced time to surgery within a 'fast track' pathway for periampullary malignancy is associated with an increased rate of pancreatoduodenectomy. HPB (Oxford) 2017;19:713-20.

8 National Institute for Health and Care Excellence. Pancreatic cancer in adults: diagnosis and management: NICE guideline [NG85]. NICE, 2018. www.nice.org.uk/guidance/ng85/chapter/ Recommendations\#managing-resectable-and-borderlineresectable-pancreatic-cancer
9 National Institute for Health and Care Excellence. A model of fasttrack surgery for pancreatic cancer to implement the NICE guideline recommendations for the management of operable patients. NICE, 2019. www.nice.org.uk/sharedlearning/a-model-of-fast-tracksurgery-for-pancreatic-cancer-to-implement-the-nice-guidelinerecommendations-for-the-management-of-operable-patients

10 Oken MM, Creech RH, Tormey DC et al. Toxicity and response criteria of the Eastern Cooperative Oncology Group. Am J Clin Oncol 1982;5:649-55.

11 Roberts KJ. Promoting Innovative Practice: Fast-track surgery for pancreatic cancer reduces time to treatment, complications and increases the number of people undergoing successful surgery. Pancreatic Cancer UK, 2019.

12 Rubin HJ, Rubin IS. Qualitative interviewing: the art of hearing data. SAGE Publications, 2012.

13 Kallio H, Pietila AM, Johnson M, Kangasniemi M. Systematic methodological review: developing a framework for a qualitative semistructured interview guide. J Adv Nurs 2016;72:2954-65.

14 Braun V, Clarke V. Using thematic analysis in psychology. Qual Res Psychol 2006:3:77-101.

15 Oba A, Ho F, Bao QR et al. Neoadjuvant treatment in pancreatic cancer. Front Oncol 2020;10:245.

16 Best A, Greenhalgh T, Lewis $S$ et al. Large-system transformation in health care: a realist review. Milbank Q 2012;90:421-56.

17 RICOCHET Study Group, West Midlands Research Collaborative. Receipt of Curative Resection or Palliative Care for Hepatopancreaticobiliary Tumours (RICOCHET): Protocol for a nationwide collaborative observational study. JMIR Res Protoc 2019;8:e13566.

18 Oppong SH. The problem of sampling in qualitative research. Asian J Manag Sci 2013;2:202-10.

Address for correspondence: Basil Yannoulias, College of Medical and Dental Sciences, University of Birmingham, Edgbaston, Birmingham B15 2TT, UK.

Email:vasyannoulias@gmail.com

Twitter: @yannoulias 